\title{
La prévision statistique du champ de précipitations
}

\author{
Statistical forecasting of the field of precipitation
}

\author{
G. Der Megreditchian, M. Nuret, $\mathbf{M}^{\mathrm{me}}$ M. C. Rullière et R. Stangret \\ Météorologie Nationale
}

\section{Introduction}

La pluviométrie est un élément météorologique difficile à prévoir et les tentatives effectuées à ce jour ont concerné essentiellement un aspect purement local du problème. La variante opérationnelle de la prévision statistique des précipitations élaborée à la Météorologie Nationale [1], qui comportait initialement la prévision des précipitations aux 6 centres régionaux, bien qu'en cours d'extension à 90 stations ne représente toutefois qu'une juxtaposition de 90 prévisions locales, chaque variable prédictand étant traitée séparément lors de l'élaboration du modèle prévisionnel.

Dans cet article nous abordons une approche plus globale tenant compte d'une information supplémentaire, la cohésion spatiale du champ de précipitations. Nous conservons ainsi au champ prédictand son caractère spatio-temporel.

\section{Position du problème :}

La variable prédictand est un champ météorologique décrit par un vecteur à 90 dimensions dont chaque composante représente la valeur moyenne départementale des pluies quotidiennes mesurées aux postes pluviométriques appartenant à ce département. Le fichier des données correspondantes, élaboré par le Bureau de l'Eau de la Météorologie Nationale, s'étend sur une période allant du $1^{\text {er }}$ janvier 1961 au 31 décembre 1978.

Le fichier des prédicteurs est constitué tout d'abord par les 20 champs de géopotentiel et de température aux 10 niveaux standards (Prédicteurs Synoptiques Primaires) définis aux points d'une grille $10 \times 10$ extraite du fichier PANAL, recouvrant le domaine "Europe" et qui ont servi à élaborer trente huit autres champs (Prédicteurs Synoptiques Secondaires).
Les champs prédicteurs que nous avons créés sur le fichier sont ainsi : géopotentiel, température, tourbillon absolu, advection de tourbillon absolu, advection de température, vent zonal, vent méridien, force du vent; vitesses verticales.

Ces champs sont pris aux réseaux du $12 T U(j)$ et $00 T U(j+1)$ pour expliquer la pluie au jour " $j$ ", car il faut tenir compte du décalage qui existe entre le "jour légal et le "jour de la pluie".

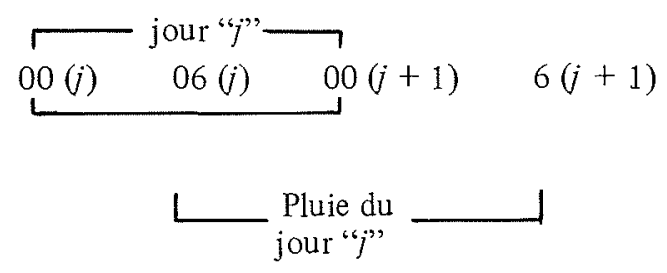

\section{Traitement statistique du fichier prédictand}

"Qui veut prévoir l'avenir doit bien connaître le passé", c'est pourquoi une étude statistique détaillée a été effectuée pour mettre en évidence le comportement statistique moyen, c'est-à-dire la climatologie du champ prédictand. Nous avons ainsi calculé les champs de valeurs moyennes et des écart-types associés aux périodes décadaires, mensuelles, saisonnières, annuelles. Des exemples sont présentés sur la figure 1.

La cohérence statistique spatiale du fichier a été étudiée par une analyse de corrélation et on présente sur la figure 2 une cartographie de certains extraits de la matrice $R Y Y(90 \times 90)$, montrant le domaine d'influence du phénomène pluie en un lieu donné.

Une autre analyse de corrélation a permis d'appréhender la mémoire temporelle du champ de pluie ; pour chaque département on a calculé la fonction d'autocorrélation temporelle de la quantité de précipitations. 
nowonroi $-\ldots j$

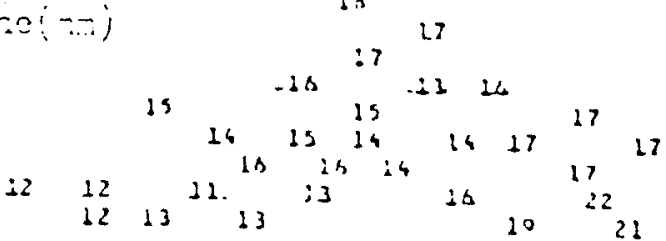

$$
\begin{aligned}
& 1212{ }_{11}^{13} \text { i2 } 13 \text { 13 } 17 \mathrm{is}^{23} \\
& \text { 10. } 10{ }_{11}^{13} \quad 19 \quad 17 \quad 23
\end{aligned}
$$

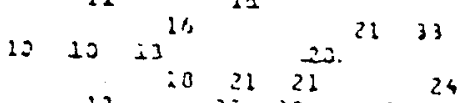

$$
\begin{aligned}
& 11 \quad 12 \quad 20 \quad 212^{21} 0^{21} \quad 22 \quad 24
\end{aligned}
$$

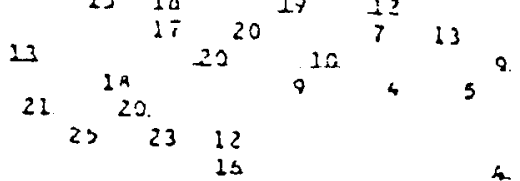

$\cos i-\operatorname{tinc}(\cdots . \cdots) \quad 22$ is

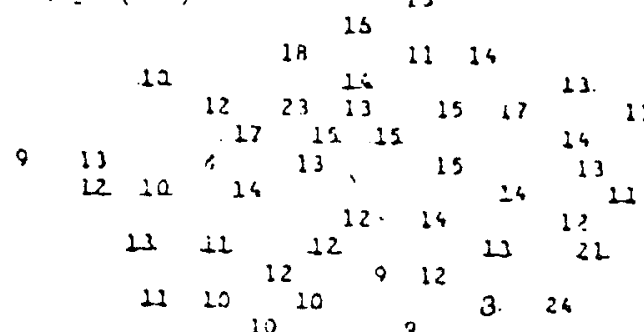

$9812^{36 .} i^{4} 15 \quad 22$

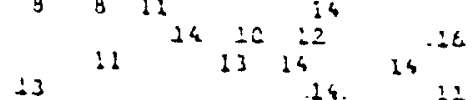

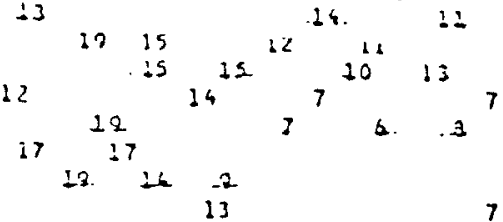

Fig. 1.1 - Première décade du mois de juillet.

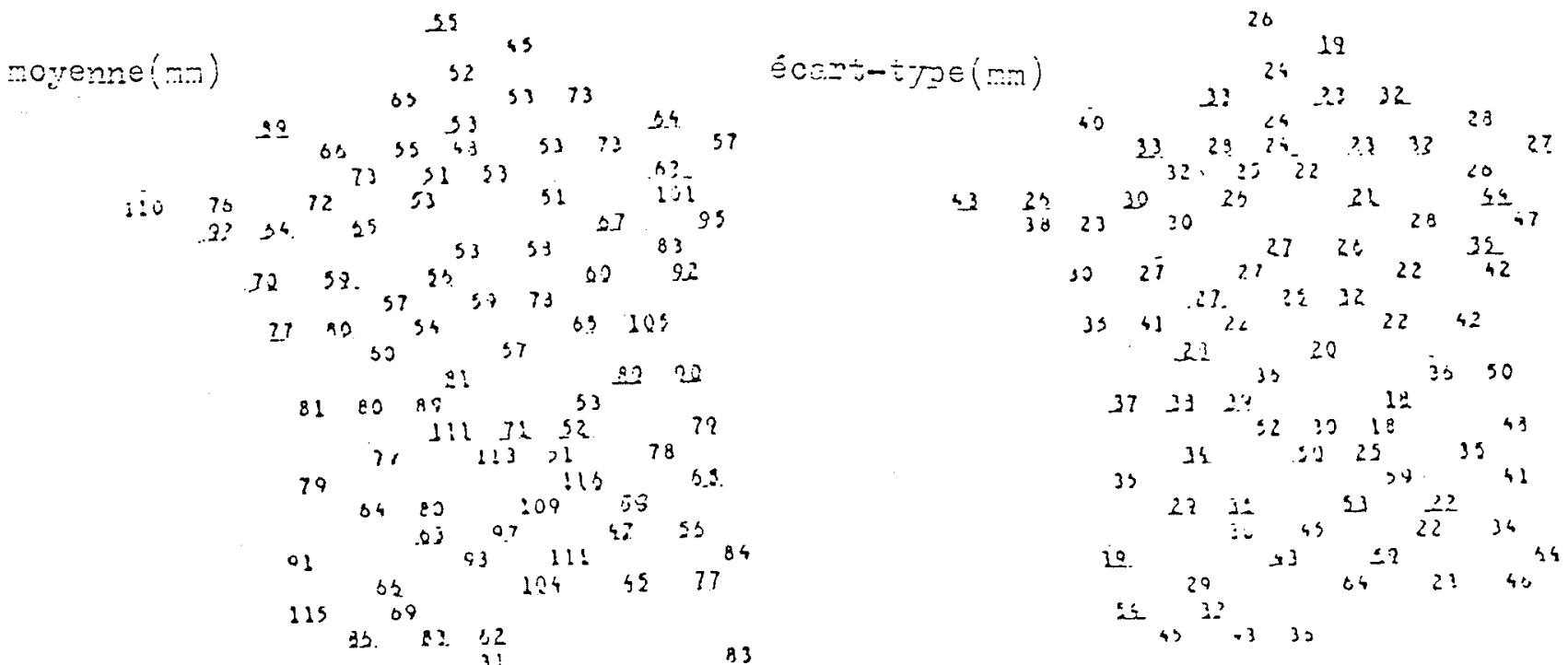

Fig. 1.2 - Mois de janvier.
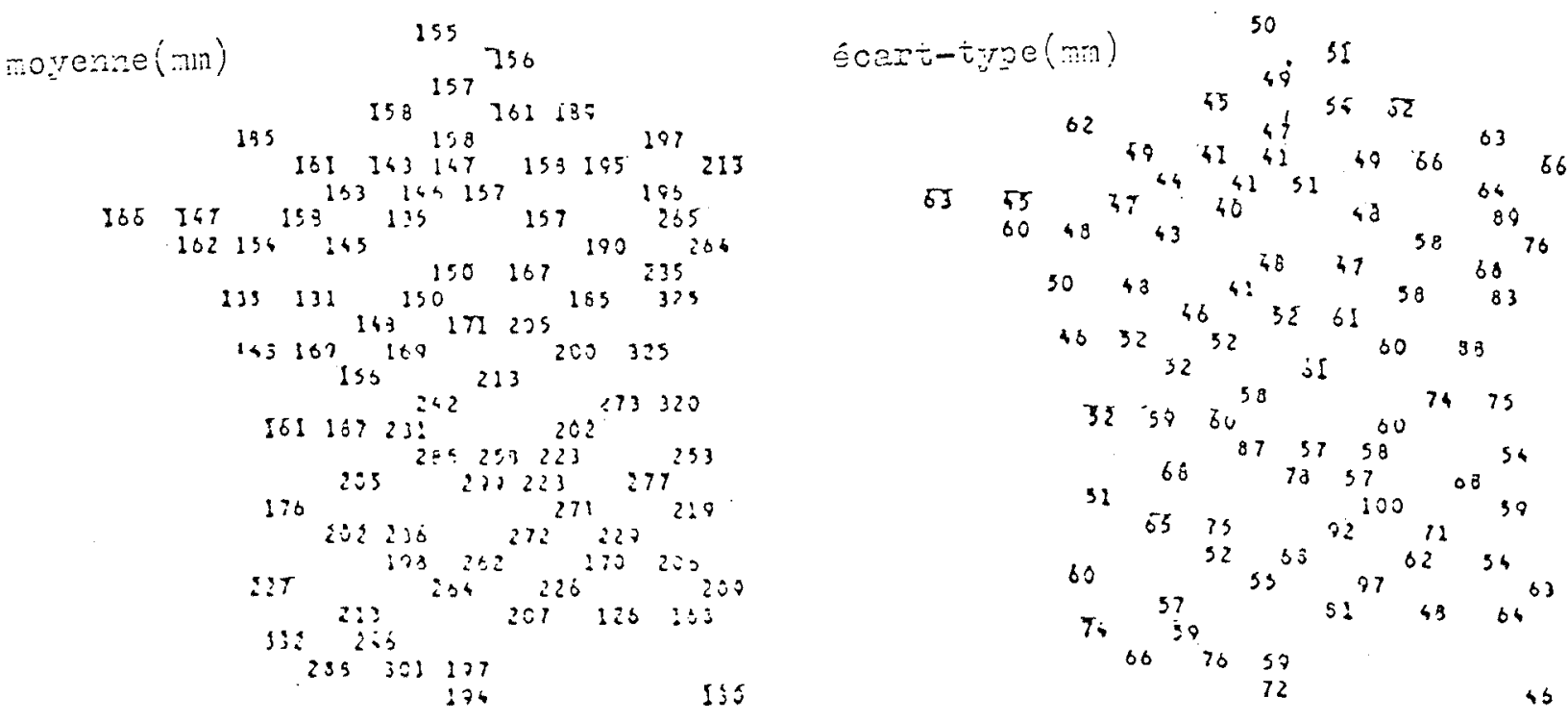

Fig. 1.3 - Saison printemps. 


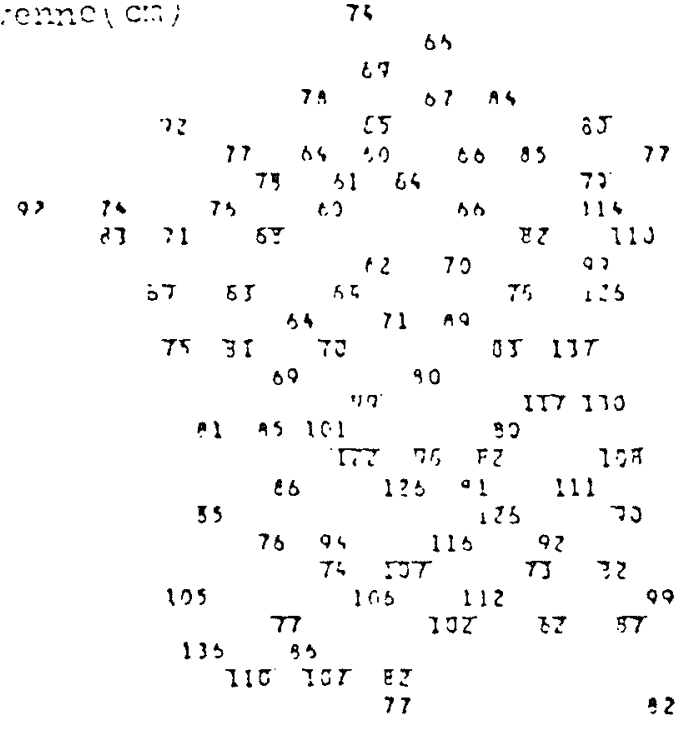

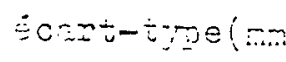$$
\text { tope( }
$$$$
13
$$$$
21212
$$$$
12
$$

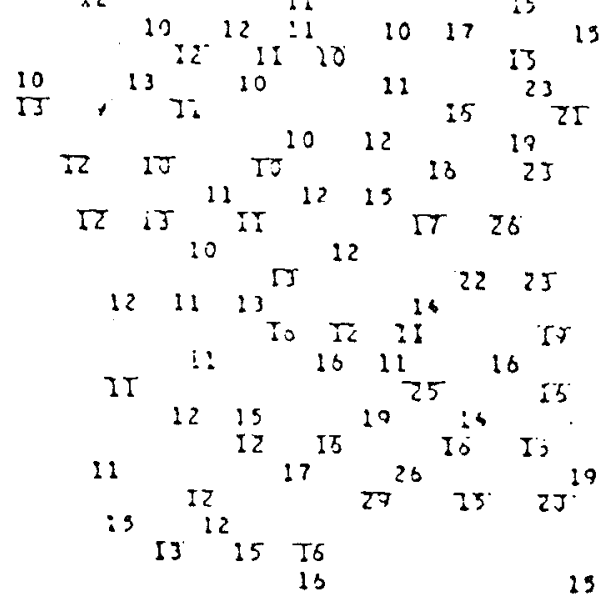

Fig. 1.4 - Année

Figure 1 - Caractérisation Statistique (valeurs moyennes et écarts-types) de la quantité de précipitations associée à différentes périodes : décadaire (Fig. 1.1), mensuelle (Fig. 1.2) saisonnière (Fig. 1.3), annuelle (Fig. 1.4).

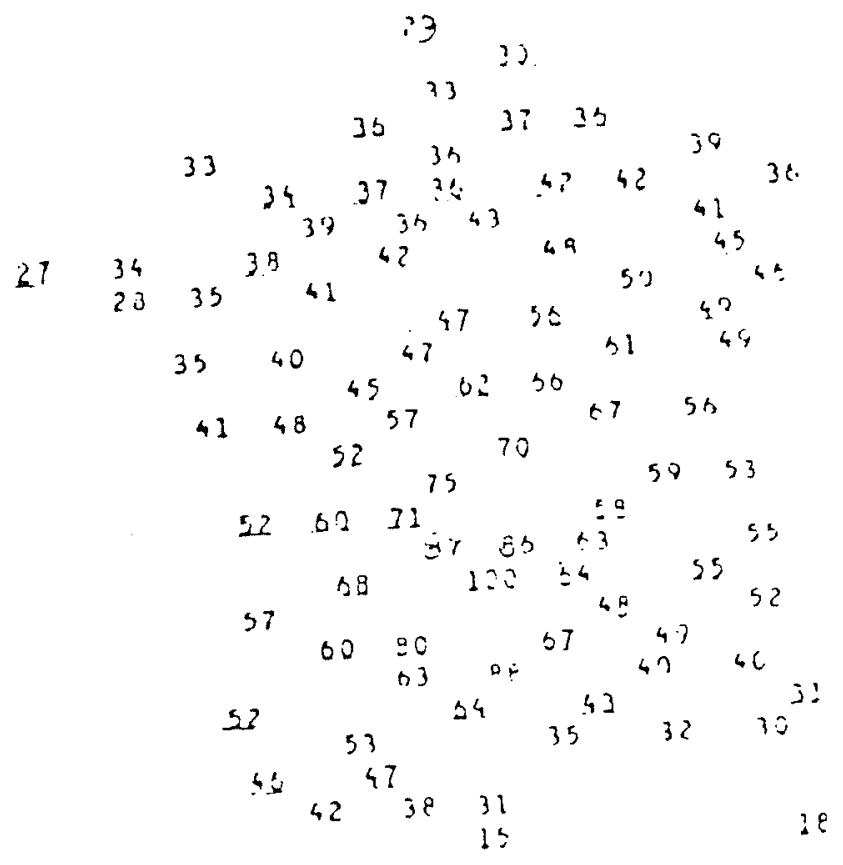

$$
\begin{aligned}
& 50
\end{aligned}
$$

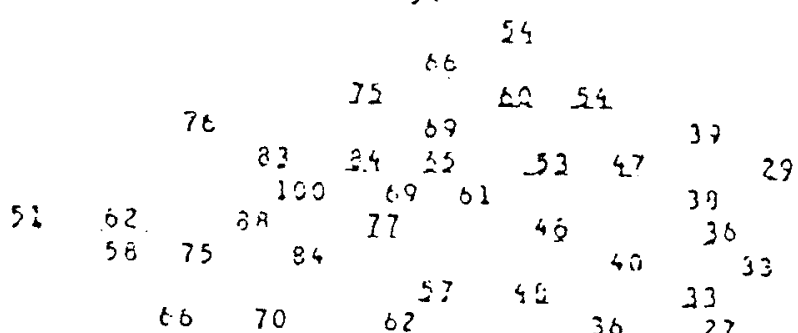

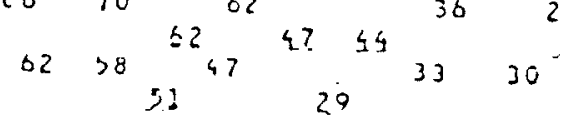

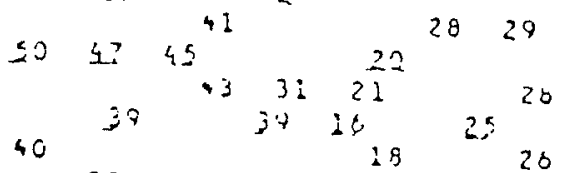

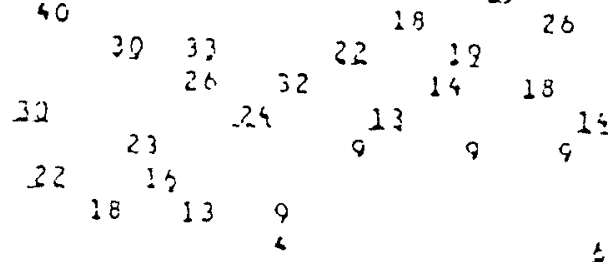

Figure 2 - Cartographie des corrélations entre les valeurs de la pluie quotidienne sur un département (Fig。2.1 Cantal; Fig. 2.2 Orne) et sur les autres.

Une analyse de l'homogénéité du fichier (spatiale et temporelle) a été effectuée par une méthode de Classification Hiérarchique Automatique utilisant différentes métriques (Minkowski, Hamming, Khi-deux). Nous avons pu ainsi mettre en évidence des zones au comportement pluviométrique similaire (régionalisation) et des périodes pour lesquelles les caractéristiques statistiques du prédictand restent stables (saisonnalité).

Dans cet article on présentera l'étude effectuée sur la période "hiver" de septembre à février.

\section{Le modèle prévisionnel}

La méthode de prévision employée est celle dite de "prévision parfaite" pour laquelle la valeur de $Y$ prévues au temps $t+\tau$ s'obtient à partir des liaisons synchrones entre champs prédicteurs et champ prédictand. Les champs prédicteurs $x(t+\tau)$ ne sont évidemment pas connus à l'instant $t$ où l'on fait la prévision, de sorte qu'on les remplace par les valeurs $\tilde{x}(t+\tau)$ prévues par le modèle dynamique. On devrait alors en toute logique 


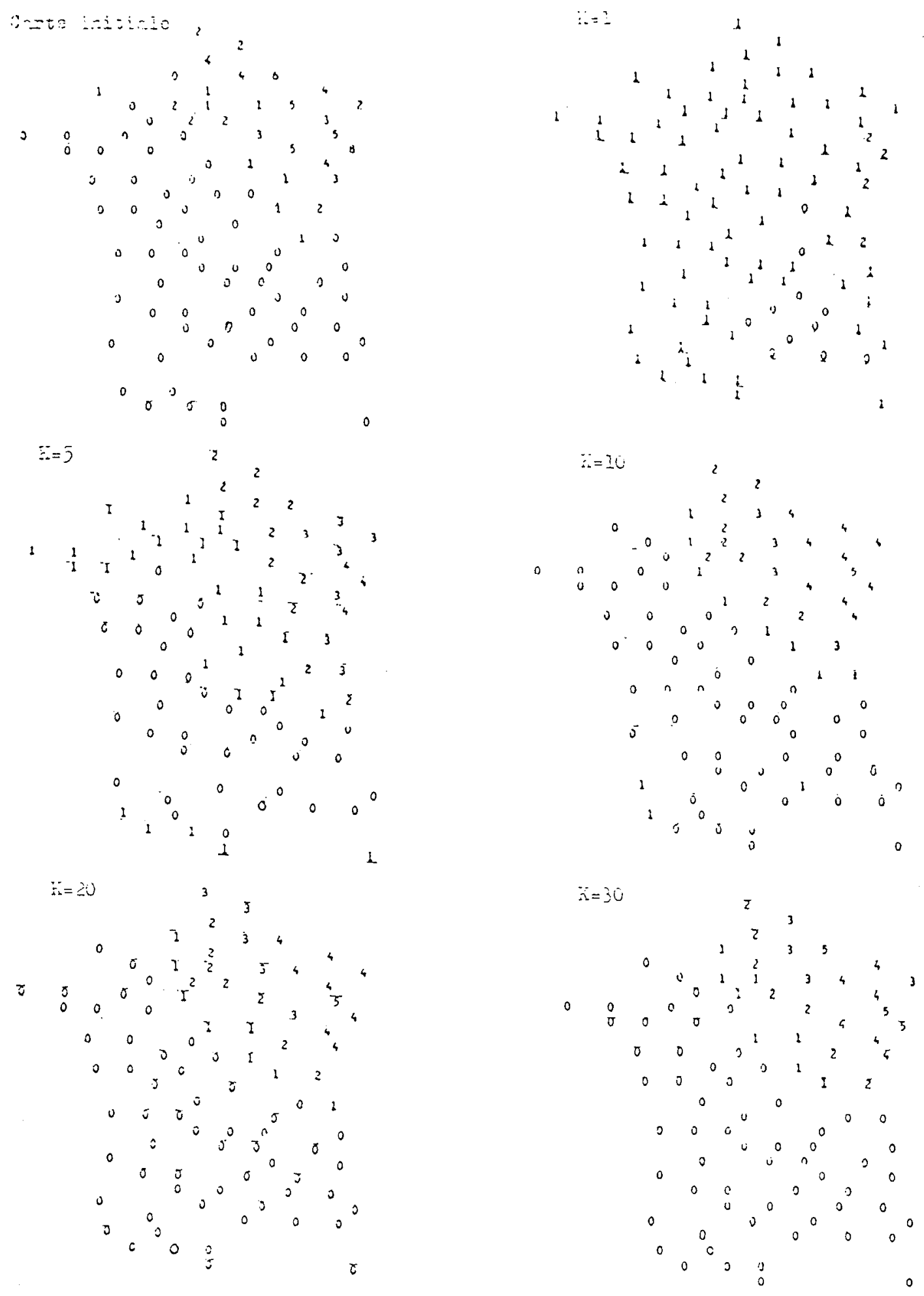

Figure 3.1 - Exemple de reconstitution du champ prédictand $Y$ avec $K$ composantes principales. 


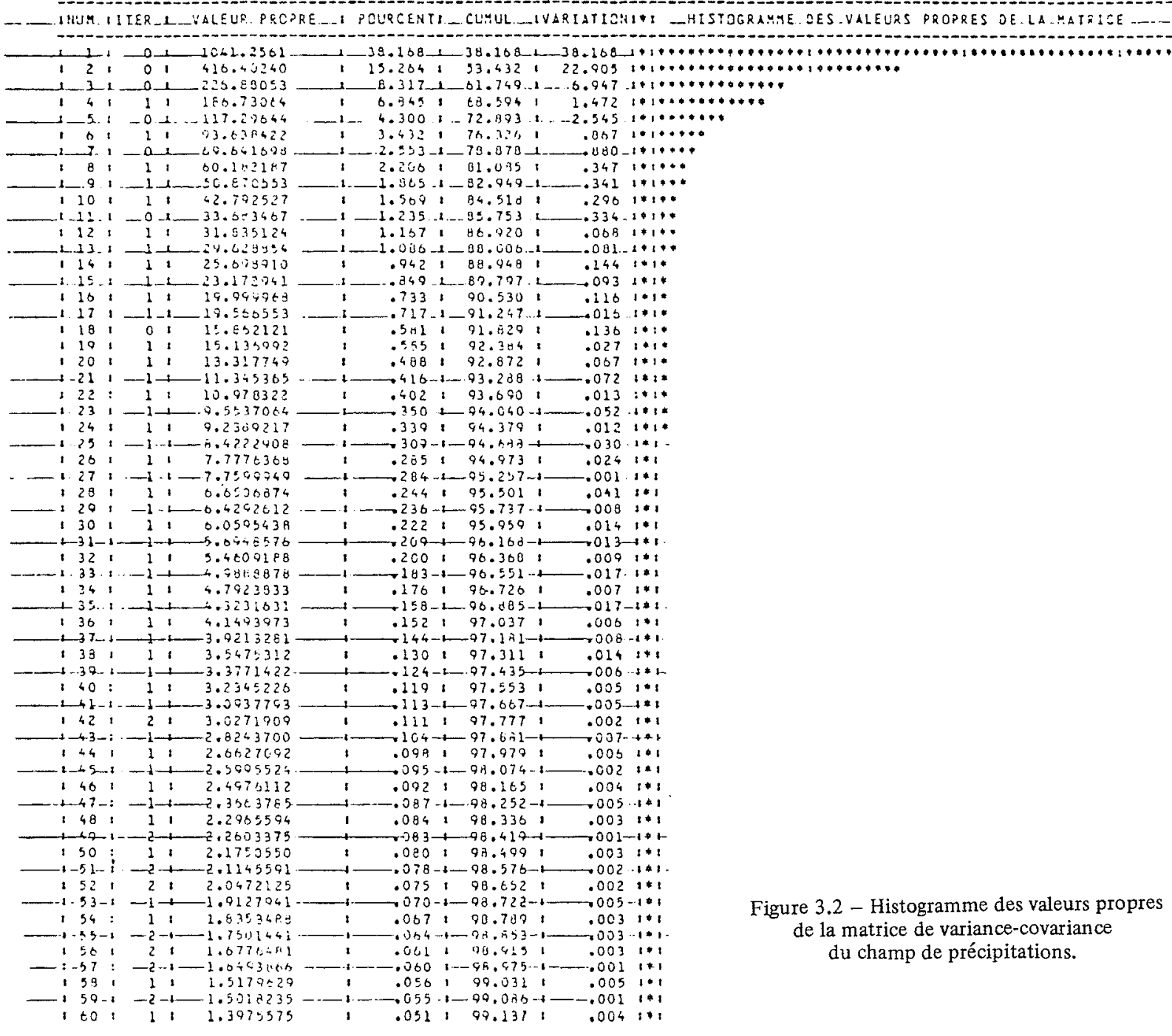

utiliser dans l'algorithme prévisionnel les corrélations synchrones correspondantes prédicteurs-prédictand $R_{x y} \tilde{y}$. Le fichier des $\widetilde{X}$ constitué à ce jour étant trop petit (2 ans) nous avons substitué aux corrélations $R_{x \tilde{y}}$ les corrélations $R_{x y}$ entre champs prédicteurs rééllement observés et champ prédictand que l'on peut calculer sur l'ensemble du fichier d'apprentissage (14 ans).

La validité de cette substitution sera d'autant mieux confirmée que la qualité de la prévision du modèle dynamique sera bonne. Cela fait que pour différentes échéances $\tau$ de la prévision, on utilise les mêmes équations avec une qualité se dégradant avec celle du modèle dynamique.

Le principe de la prévision est le suivant : on décompose le champ prédictand à l'aide d'une Analyse en Composantes Principales afin d'éliminer les fluctuations ponctuelles de faible intensité tout à fait imprévisibles, puis on utilise les champs prédicteurs les plus informatifs pour prévoir chacune des Composantes Principales retenues et enfin on reconstitue le champ prédictand à l'aide des Composantes Principales prévues.

Sur la figure 3.1 on présente un exemple concret de reconstitution du champ de précipitations pour un jour d'été avec respectivement $1,5,10,20,30$ composantes principales. On notera ici la bonne qualité de la condensation de ce champ, tout à fait conforme à la croissance rapide du pourcentage cumulé $Q_{K}[Y]$ de variance expliquée par les $K$ premières composantes principales :

$$
Q_{K}[Y]=\sum_{j=1}^{k} \frac{\lambda_{j}\left[V_{y y}\right]}{\operatorname{tr}\left[V_{y y}\right]}
$$

(où $\lambda_{j}\left[V_{y y}\right]$ désigne la $j^{\text {ème }}$ valeur propre de $V_{y y}$ et $\operatorname{tr}\left[V_{\nu y}\right]$ la trace de cette matrice) que révèle l'histogramme des valeurs propres (Fig. 3.2); on vérifie ainsi que $Q_{1}(Y)=0,38 ; Q_{2}(Y)=0,53 ; Q_{10}(Y)=0,84$; $Q_{30}(Y)=0,96$. Ceci est très satisfaisant. Les trente premières composantes principales que nous avons retenues restituent une image suffisamment fidèle du champ initial.

Le schéma directeur du modèle prévisionnel matérialisant le principe adopté est présenté sur la figure 4 .

\section{La sélection des prédicteurs}

Nous disposons ainsi de 108 champs prédicteurs potentiels (soit 10800 variables individuelles) apportant une certaine information redondante au sujet du champ 
ENTREE

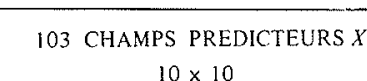

$10 \times 10$

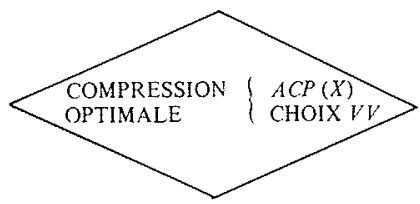

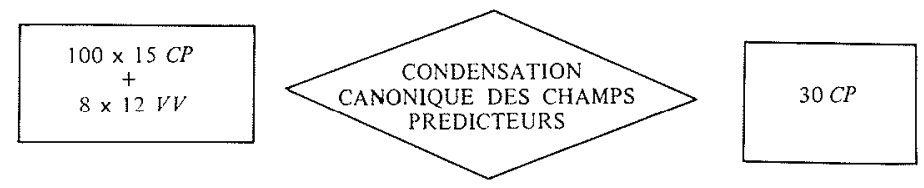
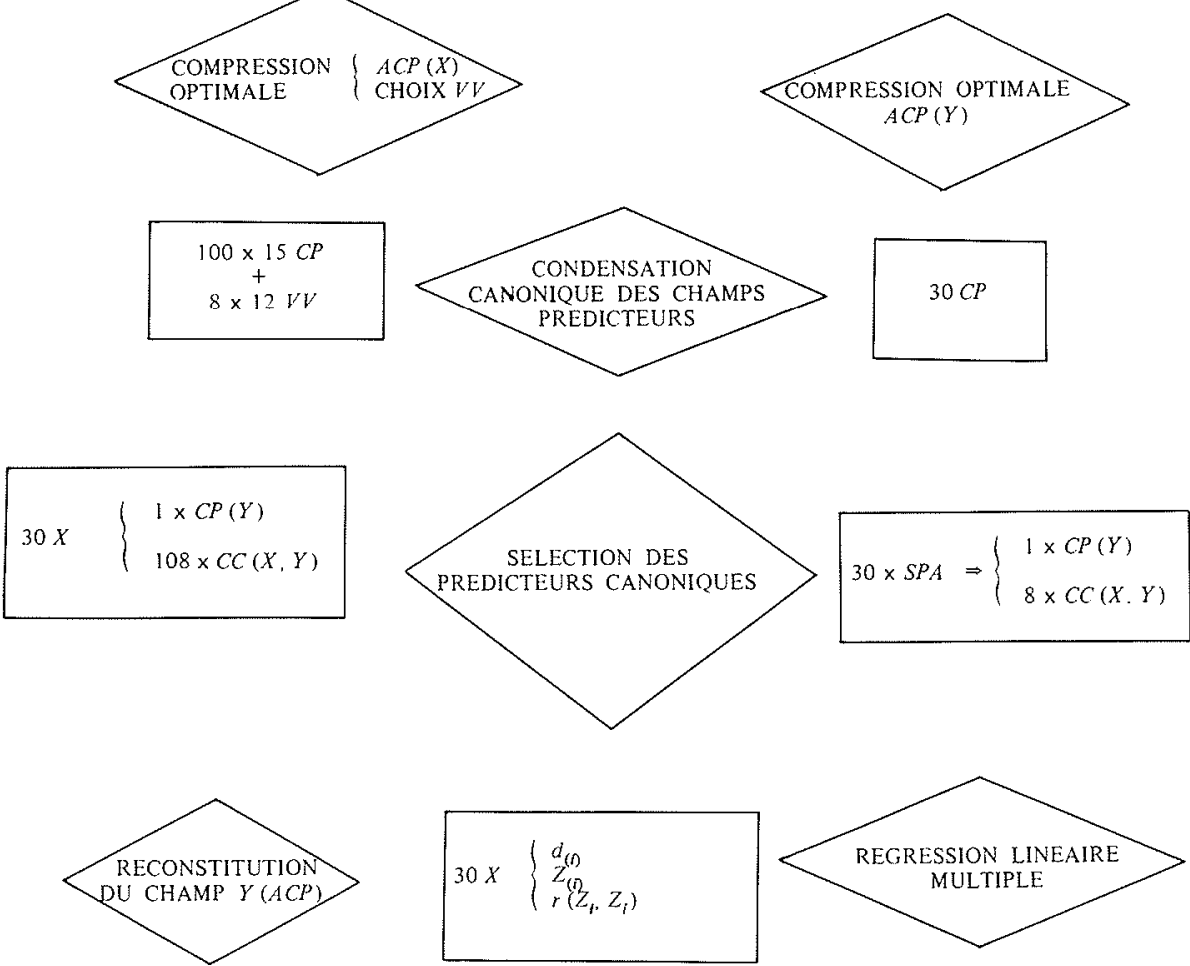

SORTIE

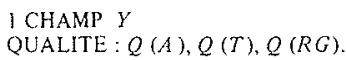

Figure 4 - Organigramme du modèle prévisionnel : $X$ champ prédicteur ; $Y$ champ prédictand; $A C P$ analyse en composantes principales ; $C P$ composantes principales; $C C$ composantes canoniques; $V V$ vitesses verticales; " $\sim$ "valeur prévue; $a$ vecteur des coefficients de régression; $Q$ qualité de la prévision; $Q(A)$ sur fichier d'apprentissage, $Q(T)$ sur fichier test, $Q(R G)$ par reconnaissance glissante.

de précipitations. C'est pourquoi un tri entre information utile et parasite est absolument nécessaire pour éliminer cette redondance. Ce tri a été réalisé en conservant aux prédicteurs leur caractère de champ météorologique. Nous n'avons pas appliqué une procédure de sélection aux 10800 variables, non pas par impossibilité technique, mais parce que les résultats auraient été absolument non robustes sur fichier test.

La sélection des prédicteurs est habituellement effectuée sur des variables scalaires, de sorte que nous avons tout d'abord élaboré pour chaque Composante Principale du prédictand de nouveaux prédicteurs scalaires canoniques associés à chaque champ prédicteur et qui recèlent toute l'information (au sens d'une opération linéaire) contenue dans ce champ au sujet de la variable à expliquer.

Nous avons ensuite effectué une Sélection Progressive Ascendante des 108 variables canoniques ainsi obtenues pour chaque composante principale du prédictand.

La nature des prédicteurs les plus informatifs se dégage nettement de la figure 5 montrant la fréquence d'apparition de chaque champ; parmi les meilleurs : les vitesses verticales $(21 \%)$, l'advection de tourbillon absolue $(19 \%)$ et la température (18\%).

\begin{tabular}{|l|c|c|}
\hline \multicolumn{1}{|c|}{ Prédicteurs } & $\begin{array}{c}\text { Nombre } \\
\text { d'apparitions }\end{array}$ & $\begin{array}{c}\text { Fréquence } \\
\text { de sortie }\end{array}$ \\
\hline Température & 43 & $18 \%$ \\
\hline Geopotentiel & 20 & $8 \%$ \\
\hline Vent méridien & 15 & $6 \%$ \\
\hline Vent zonal & 5 & $2 \%$ \\
\hline Tourbillon absolu & 6 & $3 \%$ \\
\hline Advection de tourbillon absolu & 42 & $18 \%$ \\
\hline Advection de température & 32 & $13 \%$ \\
\hline Force du vent & 27 & $11 \%$ \\
\hline Vitesse verticale & 50 & $21 \%$ \\
\hline
\end{tabular}

Figure 5 - Fréquence relative d'apparition d'un prédicteur lors de la sélection. 


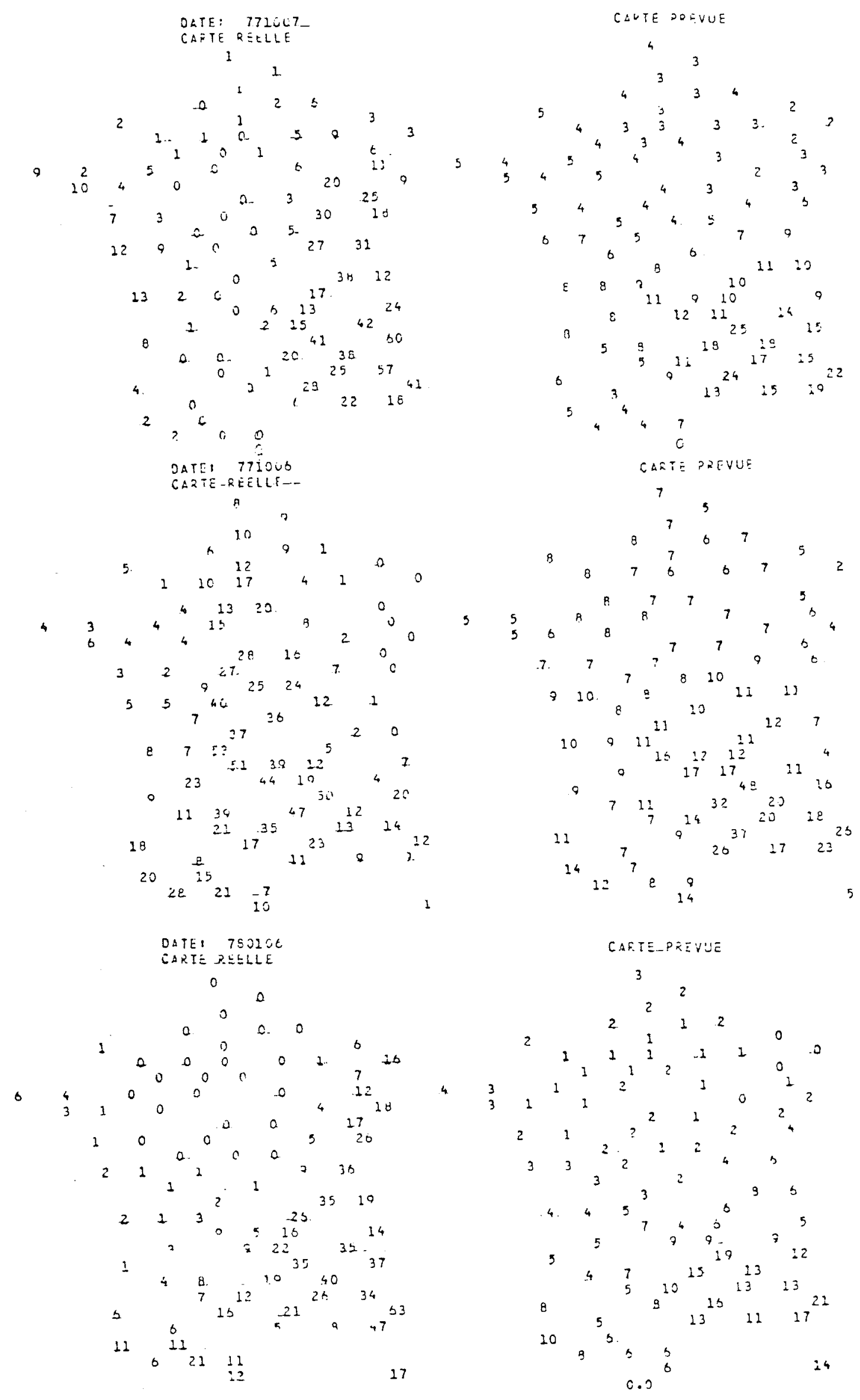

Figure 6 - Exemples de prévision đe la carte des précipitations réalisée sur le fichier test. 
En ce qui concerne le nombre $K$ de prédicteurs retenus, nous avons choisi intentionnellement une valeur commune, quelque peu sous-estimée, afin d'améliorer la robustesse du schéma prévisionnel lors du passage fichier d'apprentissage-fichier test. Nous avons ainsi décidé de garder huit prédicteurs bien que parfois nous aurions pu en garder $9,10,11$ ou 12. La stabilité acceptable de la qualité de la prévision que nous avons observée confirme le bien-fondé de ce choix.

\section{Equations prévisionnelles}

Pour chaque composante principale, nous avons appliqué la méthode de Regression Linéaire Multiple afin d'élaborer les équations prévisionnelles permettant de relier les 8 prédicteurs canoniques sélectionnés au prédictand correspondant.

Pour étudier les fortes précipitations, nous avons également testé une nouvelle variante de Régression Minimax dont le principe est, comme son nom l'indique, de minimiser l'erreur maximum généralement associée aux pluies exceptionnelles.

Dans une application pratique, le choix entre la valeur prévue de y par ces deux méthodes peut-être fait par une Analyse Discriminante [2].

A partir des valeurs prévues des 30 premières composantes principales on utilise la formule de reconstitution de l'ACP pour rétablir le champ prévu des précipitations.

\section{Qualité du modèle prévisionnel}

On présente sur la figure 6 quelques exemples de prévision concrète sur lesquels on remarque, malgré une certaine atténuation de l'intensité des précipitations (due au principe même de la régression) que l'on retrouve bien la configuration spatiale des pluies sur la France. Le régime des précipitations est donc correctement perçu.

Une estimation globale de la qualité a été réalisée aussi bien sur le fichier d'apprentissage (du 1.1.63 au 2.2.77) que sur le fichier test (du 1.9.77 au 28.2.78). L'indice de la qualité retenu est la carte des corrélations $r(y i, \tilde{y} i)$ entre la valeur des précipitations réellement observée pour le département " $i$ " et sa valeur prévue pour ce même département (Fig. 7).

On constate, d'une part que les valeurs des coefficients de corrélation sont assez élevées sur le fichier d'apprentissage et que la dégradation sur le fichier test est tout à fait raisonnable pour cet élément météorologique.

On remarque également pour quelques départements (11 et 66) que l'influence locale très spécifique prédomine sur le caractère synoptique des interrelations spatiales de sorte que la qualité dans ces départements est plus faible sur les deux fichiers.

Par contre, l'organisation spatiale notoire des coefficients de corrélation correspond bien à certaines idées météorologiques communément admises (valeurs élevées sur le littoral, prédominance des régimes d’Ouest).

\section{Conclusion}

Le modèle de prévision des précipitations quotidiennes départementales permet l'élaboration d'une carte synthétique des précipitations prévues sur la France, pouvant intéresser de nombreux utilisateurs (Agriculture, EDF) plus concernés par la distribution spatiale des précipitations que par la connaissance des valeurs ponctuelles en un lieu donné.

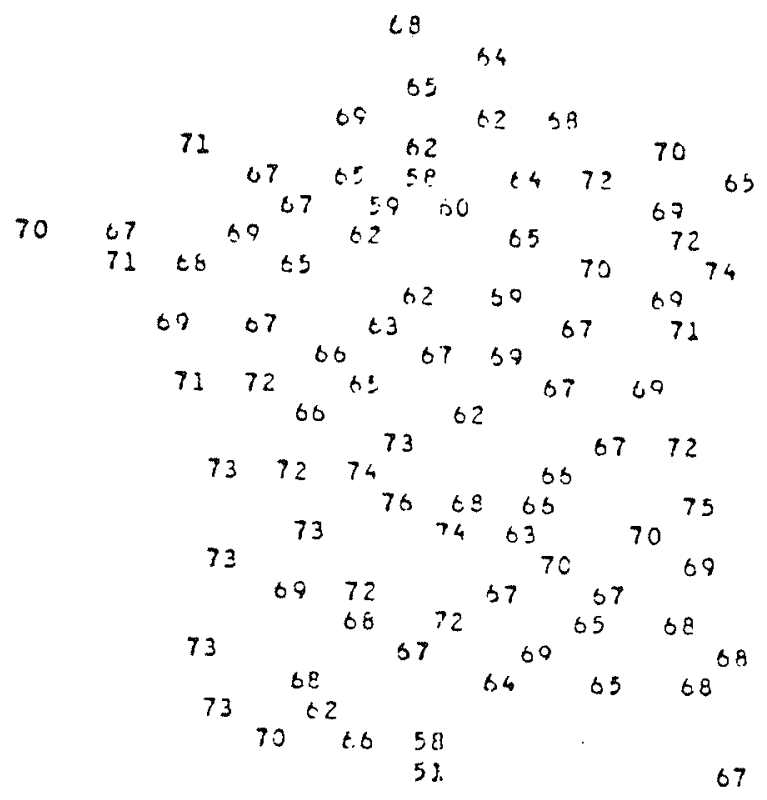

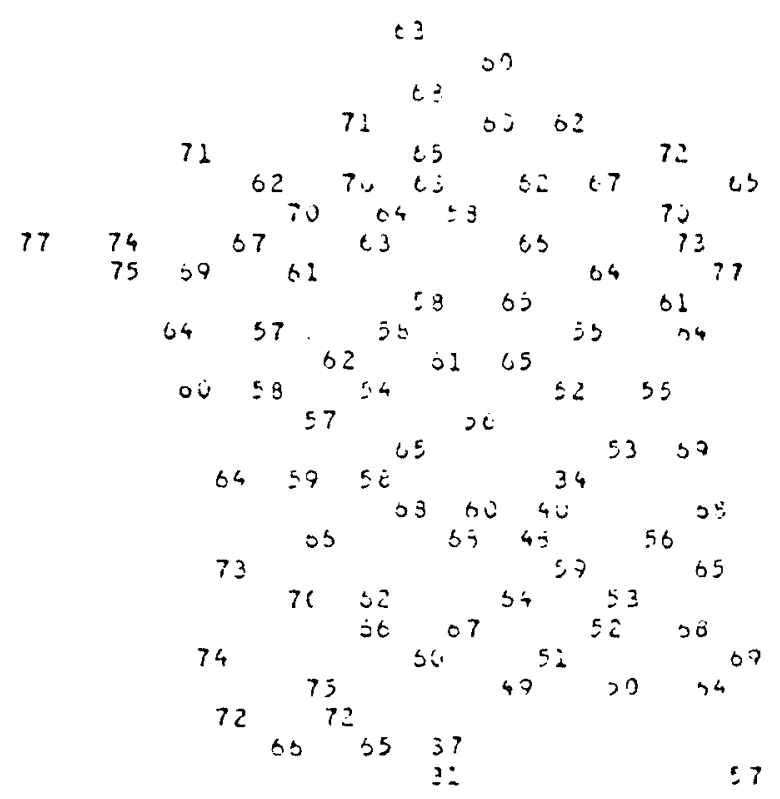

FICHIER CAAPPFEATISSAQE

Figure 7 - Corrélation entre la pluie réelle et la pluie prénue pour chaque département. 
La qualité obtenue [3] semble suffisamment bonne pour que l'on puisse envisager l'élaboration d'une variante opérationnelle de ce modèle.

Divers développements sont également envisagés, sur le plan méthodologique par l'élaboration de nouveaux algorithmes prévisionnels et par l'introduction de nouveaux prédicteurs, sur le plan météorologique par un raffinement du découpage du fichier (saisons hydrologiques et régions climatiques homogènes).

\section{Bibliographie}

[1]JAVELLE, DER MEGREDITCHIAN, CALVEYRAC MUSSON-GENON, STANGRET, VEYSSEIRE. - Statistical and Dynamical adaptation of the forecasts given by the French 8.E. model.

[2] DER MEGREDITCHIAN G. - La précision statistique des phénomènes météorologiques. Note EERM. N 100, Paris 1981.

[3] NURET $M_{*}$, STANGRET R. - Approche statistique de la prévision d'un champ de précipitations sur la France. Thèse de fin d'Etudes E.N.M.

\section{Discussion}

Le Président. - Merci M. STANGRET. Ce travail, résultat de la collaboration de deux équipes de la Météorologic Nationale, a l'intérêt de traiter de façon globale l'aspect spatio-temporel du champ des précipitations en associant des prédicteurs issus d'un modèle dynamique de prévision et un champ de précipitations issus d'une analy se statistique des données au sol.

Il n'est pas étonnant de constater que les prédicteurs principaux sont la vitesse verticale, l'advection de tourbillon absolu et la température.

La comparaison des champs de géopotentiels, de températures ou de précipitations simulés et observés n'est pas évidente. En particulier les événements extrêmes sont généralement mal reconstitués, mais il est important que vous ayiez pu restituer les grands traits du champ de précipitations.

M. GIRAUD (Ecole Nationale de la Météorologie). - Je souhaiterais que vous précisiez pourquoi vous n'avez pas tenu compte de l'humidité, facteur pourtant essentiel des précipitations.

M. STANGRET. - L'humidité n'est pas mesurée, et n'est fournie en sortie des modèles dynamiques que pour les quatre dernières années, alors que nous travaillons, avec les autres champs de prédicteurs, sur 20 années.

Nous avons préféré une méthode dite" "de prévisions parfaites" plutôt que les méthodes de "sorties de modèle", afin de ne pas dépendre des modifications des modèles dynamiques de la Météorologie Nationale, et parce que les sorties de modèles concernent des historiques encore insuffisants.

M. DER MEGREDITCHIAN. - Par la méthode de prévision parfaite, nous pouvons en entrée utiliser les sortics d'un modèle dynamique quelle que soit l'échéance de prévision : 24 heures, 48 heures, etc. C'est la qualité du modèle dynamique qui est prépondérante en l'occurrence.

M. THIERY (BRGM). - Pourquoi travaillez-vous sur les pluies journalières sans transformation ? Quelle signification accordez-vous à des corrélations linéaires cntres des variables de distributions extrêmement dissymétriques?

M. DER MEGREDITCHIAN. - On a hésité entre plusieurs transformations (racine carrée, cubique, transformation normalisante, etc.). Il fallait commencer par quelque chose et ceci n'est qu'une première approche.

M. STANGRET. - Nous travaillons sur des moyennes départementales. On élimine ainsi les pluies d'orages localisées. Ceci permet une analyse en composantes principales correctes (38\% pour la première composante). Les valeurs moyennes ne répondent pas à tous les besoins, mais à la plupart.

M. DENEAU (EDF-DTG). - Le département est une unité artificielle, et le lissage introduit des erreurs d'autant plus grandes que les particularités locales sont plus importantes, c'est-à-dire surtout dans les régions montagneuses du Sud de la France.
La reconstitution de la pluie à partir de 30 composantes principales est-elle stable ? Les dernières composantes, représentant moins de $1 \%$ de la variance, ont-elles un sens ?

Le Président. - On constate dans l'exemple présenté que les résultats sont aussi bons avec 10 composantes.

M. STANGRET. - Le choix de 30 composantes est en effet arbitraire.

M. DER MEGREDITCHIAN. - Le département est une unité artificielle, mais c'est un domaine de grandeur à peu près homogène pour lequel les moyennes sont directement accessibles au Bureau de l'Eau. Par ailleurs, un champ ainsi moyenné se prêto bien à l'analyse en composantes principales et on peut considérer que l'on a bien représen té la répartition de ce champ moyen. Les valeurs extrêmes ont été traitées par une méthode de régression minimax permettant de réduire les grandes erreurs. Cette réduction est opérée au désavantage de la qualité de prévision moyennée. Il est nécessaire d'utiliser une procédure mixte de régression habituelle et de régression minimax.

Des essais ont eté faits pour prévoir des modalités : absence de pluie, faible pluie, forte pluie. Pour une séparation en 3 classes, le critère de sélection des prédicteurs a été un écart normé entre les valeurs des classes (comme pour 2 classes), pondéré par les tailles relatives des classes et modifié par un critère d'équilatéralité qui donne une idée des différenciations entre les couples de classes (on cherche à séparer les classes de façon homogène). Ceci permet de déterminer les scuils de classements optimaux.

M. DUBAND. - Je voudrais une confirmation : vous avez recalculé les champs de vitesse verticale sur 20 ans, et vous n'avez pas imposé de structure spatiale sur ce champ.

M. STANGRET. - Nous sommes partis du fichier banal, comportant 20 champs pour calculer des champs synoptiques secondaires et des vitesses verticales. Nous considérons, avec M. ROUSSEAU, que les composantes du champ vertical sont moins informatives que les 12 valeurs au-dessus de la France que nous avons prises en compte, sans cohésion spatiale. Ce qu'on essaie de saisir, c'est le type de situation météorologique, c'est-à-dire la structure du champ dynamique plutôt que la famille des géopotenticls.

Le Président. - Je pense que ces méthodes d'exploitation des champs dynamiques ont un intérêt capital pour la prévision météorologique. Il faut passer de ces champs aux paramètres climatiques qui intéressent les utilisateurs hydrologues en particulier. Une telle méthodologie, indépendante de la nature du modèle dynamique utilisé, est bien adaptée à l'évolution nécessaire des modèles dynamiques à l'amélioration desquels M. ROUSSEAU travaille avec son équipe de la Météorologie Nationale.

Je remercie M. STANGRET et M. DER MEGREDITCHIAN des précisions apportées. 